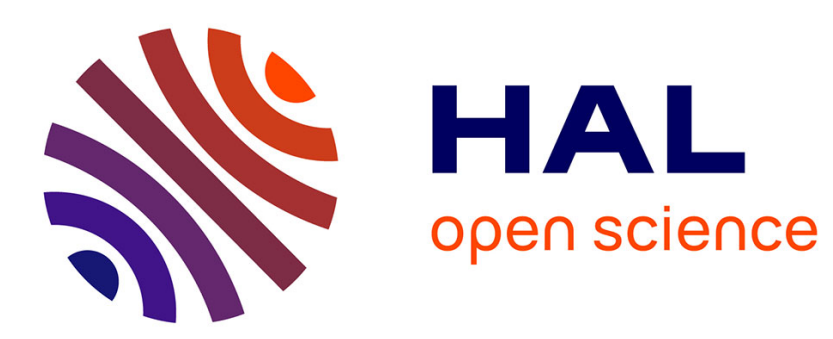

\title{
Investigation of electrical and thermal properties of carbon nanotube interconnects
}

\author{
Aida Todri-Sanial
}

\section{To cite this version:}

Aida Todri-Sanial. Investigation of electrical and thermal properties of carbon nanotube interconnects. PATMOS: Power and Timing Modeling, Optimization and Simulation, Sep 2016, Brême, Zimbabwe. pp.25-32, 10.1109/PATMOS.2016.7833421 . lirmm-01457289

\section{HAL Id: lirmm-01457289 \\ https://hal-lirmm.ccsd.cnrs.fr/lirmm-01457289}

Submitted on 6 Feb 2017

HAL is a multi-disciplinary open access archive for the deposit and dissemination of scientific research documents, whether they are published or not. The documents may come from teaching and research institutions in France or abroad, or from public or private research centers.
L'archive ouverte pluridisciplinaire HAL, est destinée au dépôt et à la diffusion de documents scientifiques de niveau recherche, publiés ou non, émanant des établissements d'enseignement et de recherche français ou étrangers, des laboratoires publics ou privés. 


\title{
Investigation of Electrical and Thermal Properties of Carbon Nanotube Interconnects
}

\author{
Aida Todri-Sanial \\ Microelectronics Department \\ CNRS-LIRMM/University of Montpellier \\ Montpellier, France 34095 \\ Email:todri@lirmm.fr
}

\begin{abstract}
Carbon nanotubes (CNTs) present themselves as a viable material for on- and off-chip interconnect material due to their unique electrical, thermal and mechanical properties. The electrothermal properties of CNTs, including high Young's modulus, resiliency and low thermal expansion coefficient offer great advantage for reliable and strong interconnects, and even more so for local and global on-chip interconnects. With aggressive scaling, on-chip interconnects contribute to power consumption and heat build-up due to their increasing parasitics with scaling which detriment overall energy efficiency of circuits. CNTs present an opportunity to address these challenges and provide solutions for reliable and energy efficient signal and power/ground interconnects. In this work, we investigate the electrical and thermal properties of CNTs based on analytical models for interconnect-level simulations. We investigate the performance of horizontally aligned CNTs as global interconnects and report on their performance.
\end{abstract}

Index Terms - carbon nanotube interconnects; electrical modeling, thermal modeling

\section{INTRODUCTION}

CNTs are a class of nanomaterials with unique mechanical, thermal and electrical properties [8]. CNTs can be classified into two types: single-wall (SWCNTs) and multi-wall (MWCNTs). SWCNTs are rolled graphitic sheets with diameters on the order of $1 \mathrm{~nm}$. MWCNTs consist of several rolled graphitic sheets nested inside each other and can have diameters as large as $100 \mathrm{~nm}$. Depending on their chirality, the CNTs can be metallic or semiconductors. Metallic CNTs (m-CNTs) are ballistic conductors, which show promise for use as interconnects in nanoelectronics. On the other hand, semiconducting CNTs (s-CNTs) have a diameter-depended band-gap and do not have surface states that need passivation, thus can be used to make devices such as diodes and transistors [11], [9], [8], [6].

Carbon nanotubes are cylindrical carbon molecules formed by one-atom thick sheets of carbon, or graphene. Carbon nanotubes, both SWCNT and MWCNT are being investigated for a variety of nanoelectronics applications because of their unique properties [8], [6], [17]. Their extraordinary large electron mean free paths and resistance to electromigration make them potential candidates for interconnects in large scale systems. During the past decade, most of research is focused on CNT growth, synthesis, modelling and simulation and characterizing contact interfaces [15], [5]. Detailed simulation for signal interconnects have been performed by [11], [9] and shown that CNTs have lower parasitics than $\mathrm{Cu}$ metal lines, however, the contact resistance between CNT-to-CNT and CNT-to-metal is large and can be detrimental for timing issues. Additionally, researchers are looking into different CNT growth techniques that are compatible with CMOS process and lab measurements indicate the potential of integrating CNTs on-chip [18], [14].

One essential and most interesting application of the nanotubes in microelectronics is as interconnects using the ballistic (without scattering) transport of electrons and the extremely high thermal conductivity along the tube axis [2]. Electronic transport in SWCNTS and MWCNTS can go over long nanotube lengths, $1 \mu \mathrm{m}$, enabling CNTs to carry very high currents (i.e. $>10^{9} \mathrm{~A} / \mathrm{cm}^{2}$ ) with essentially no heating due to nearly $1 \mathrm{D}$ electronic structure.

In literature, the comparison of copper and CNTs have been limited to signal interconnects. Investigation of global interconnects such as power and clock delivery with CNTs would also have a significant importance. It would reveal whether or not CNTs can potentially replace both signal, clock and power/ground copper interconnects. Additionally, global interconnects are most vulnerable to electromigration, it is therefore essential to assess CNTs for improving their reliability. Additionally, most of the CNT modeling and investigations are focused on their electrical properties, whereas few works exist that look into their electro-thermal modeling and properties. This work aims to investigate electrothermal properties of CNTs.

From the large body of research related to CNT analysis, mainly two groups of works can be identified. The first group of works focuses on modeling aspects of CNT inteconnects, notably [2], [8], [6], [12]. The second group of works focuses on performance comparison of CNT interconnects versus copper $(\mathrm{Cu})$ interconnects, notably as in [11], [19], [15], [6]. Complementary to these efforts, in this paper, we investigate electro-thermal properties of horizontally aligned CNTs for global interconnects such as power/ground delivery network.

The rest of this paper is organized as follows. Section II provides a detailed description of electrial properties of CNT interconnects. Section III provides description and discussions on electro-thermal modeling of CNTs where we present some electro-thermal analysis for CNTs as signal interconnects and power/ground delivery networks. Section IV concludes this 


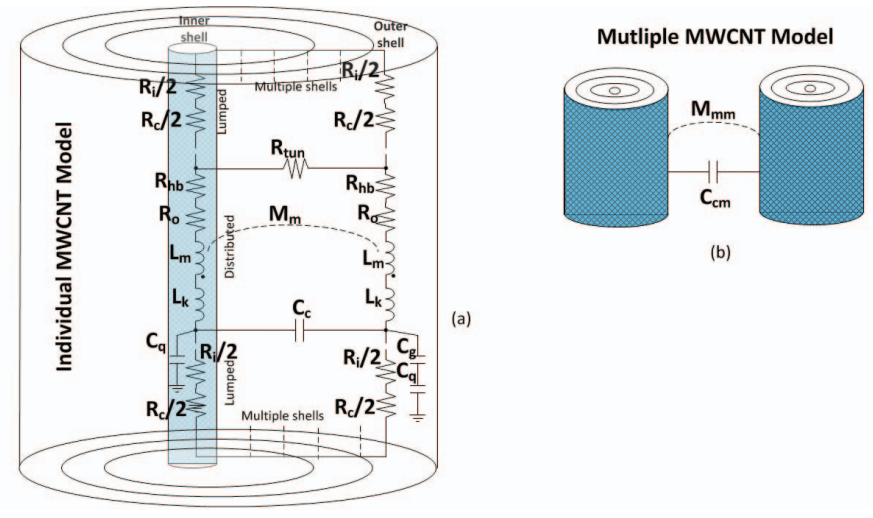

Fig. 1. (a) Circuit model of an individual MWCNT and (b) multiple MWCNTs. This is general enough model to be applicable to MWCNTs of different diameters and shell numbers. It can also be applicable to SWCNTs where the model of a single shell can be utilized [16].

paper.

\section{Electrical Modeling of CARbon Nanotubes}

There are many papers in literature that focus on CNT modeling and understanding its transport properties [2], [11], [9], [8], [6], [16]. In this section, we provide a brief description of CNT modeling that we utilize in this work. A generalized model for CNT interconnects is depicted as in Fig. 1. In Fig. 1a, the model of an individual MWCNT is shown with parasitics represent both dc conductance and high-frequency impedance i.e. inductance and capacitance effects. Multiple shells of a MWCNT are presented by the individual parasitics of each shell. Such model can also be applicable to SWCNTs where only a single shell is represented.

Each shell has a lumped ballistic resistance $\left(R_{i}\right)$, and lumped contact resistance $\left(R_{c}\right)$ due to imperfect metal-nanotube contacts. These contacts are typically constructed of Gold, Palladium, or Rhodium [8]. The nanotubes have also a distributed ohmic resistance $\left(R_{o}\right)$, which is dependent on length, $l_{b}$, and mean free path of acoustic phonon scattering $\left(\lambda_{a p}\right)$. Overall CNT resistance depends also on the applied bias voltage, $R_{h b}=$ $V_{\text {bias }} / I_{O}$, where $I_{O}$ is the maximum saturation current $\left(I_{O}\right.$ values 15 to $30 \mu \mathrm{A}$ [12]). Between shells in MWCNTs, there is also an intershell tunneling resistance $\left(R_{t u n}\right)$. As the applied bias voltage to each shell is the same, the impact of $R_{t u n}$ is relatively small. All the aforementioned ballistic, ohmic and contact resistance depend on the number of 1-D conducting channels, $N_{c}$. For metallic SWCNTs the number of conducting channels is always $N_{c}=2$ due to lattice degeneracy [2]. Whereas, for semiconducting SWCNTs and small diameter semiconducting MWCNTs, $N_{c}=0$. For any conducting shells in an MWCNT, the intrinsic resitance, $R_{i}=R_{q} / N_{c}$, where $R_{q}$ is the quanta conductance for a 1D conduction channel $\left(R_{q}=12.9 \Omega\right)$ [2]. Also, contact resistance is $R_{c}=2 R_{c o} / N_{c}$ where $R_{c o}$ is the nominal contact resistance [2]. Ohmic resistance is derived as $R_{o}=R_{q} L / N_{c} d_{s} C_{\lambda}$, where $L$ is the length of the MWCNT, $d_{s}$ is the diameter of the shell, and $C_{\lambda}$ is the acoustic phonon scattering mean free path $\left(\lambda_{a p}\right)$. Thus, the total resistance of an individual nanotube, $\left(R_{t}\right)$ can be obtained by computing resistance of each shell, $R_{\text {shell }}=R_{i}+R_{c}+R_{h b}+R_{o}$ :

$$
G_{t}=\sum_{i=0}^{N} \frac{1}{R_{\text {shell }_{i}}}=\sum_{i=0}^{N} \frac{1}{\frac{R_{f}}{N_{c}}+\frac{R_{q} L}{C_{\lambda}\left(d_{\text {in }}+i S_{a}\right) N_{c}}}
$$

where $R_{f}=R_{i}+R_{c}+R_{h b}, d_{i n}$ is the diameter of the inner shell, $S_{a}$ is the space between shells where typically $0.34 \mathrm{~nm}$ is shell thickness and $0.34 \mathrm{~nm}$ is shell-to-shell spacing and $N$ is the number of shells in MWCNT as $N=\left(d_{\text {out }}-d_{\text {in }}\right) / S_{a}$ where $d_{\text {out }}$ is the diameter of the outer shell. In a bundle of SWCNTS or MWCNTS, the total resistance can be derived as, $R_{b}=R_{t} / n_{b}$, where $n_{b}$ is the number of bundles. For example, a metal track with width, $w$ and heigh, $h$, the number of horizontally aligned nanotube bundles can be expressed as in [12]:

$$
n_{b}=P_{m}\left(n_{h} n_{w}-\left\lfloor n_{h} / 2\right\rfloor\right)
$$

where $P_{m}$ is the probability that a nanotube is metallic and usually $P_{m}=0.3$ [2], $n_{h}$ is the number of nanotubes in vertical direction as $n_{h}=\left\lfloor h / d_{\text {out }}\right\rfloor$ and $n_{w}$ is the number of nanotubes in horizontal direction as $n_{w}=\left\lfloor w / d_{\text {out }}\right\rfloor$.

The capacitance of nanotubes consists of both quantum, $C_{q}$ and electrostatic capacitances, $C_{e}$ that can impact power supply noise on power tracks. Additionally, there is coupling capacitance between: 1) conducting shells in an individual MWCNT, $C_{c}$ and 2) individual MWCNTs depending on the proximity between them, $C_{c m}$. Using Luttinger liquid theory [2], quantum capacitance can be derived as $4 e^{2} / h_{p} v_{f} \approx 193 a F / \mu m$ per conducting channel where $h_{p}$ is Planck's constant, $e$ is charge of single electron, and $v_{F}$ is Fermi velocity in graphene. Therefore, for each shell, quantum capacitance is as $C_{q}=\frac{4 e^{2}}{h_{p} v F} N_{c} L$ and the total quantum capacitance of a carbon nanotube bundle is as:

$$
C_{q_{t}}=n_{b} \sum_{i=1}^{N} C_{q_{i}}
$$

Electrostatic coupling depends on the geometry of the CNT and also the bundle density (i.e. number of bundles, $n_{b}$ ). It is shown in [15] that CNT bundles have slightly smaller electrostatic capacitance compared to $\mathrm{Cu}$ interconnects with same dimensions. Capacitance of CNT bundles would decrease slowly with increase of bundle density [2]. However, for a MWCNT, these capacitances cannot be assumed equal due to the fringing coupling effects between shells. The electrostatic capacitance of a MWCNT which is equivalent to ground capacitance from the outer shell to the ground plane, distance $y$, can be obtained as:

$$
C_{e_{t}}=\frac{2 \pi \varepsilon}{\ln \left(y / d_{\text {out }}\right)}
$$

The shell-to-shell coupling capacitance is as in [15], [11]:

$$
C_{c}=\frac{2 \pi \varepsilon}{\ln \left(d_{\text {out }} / d_{\text {in }}\right)}
$$

and coupling capacitance $C_{c m}$ between two CNT bundles with space, $s$ can expressed as:

$$
C_{c m}=\frac{2 \pi \varepsilon}{s / d_{\text {out }}}
$$


As for inductance, CNTs have both kinetic and magnetic inductance that impact power supply noise and high frequency effects on power tracks. Again, based on the Luttinger liquid theory, the kinetic inductance per conducting shell can be theoritically expressed as $L_{k}=h_{p} L / 4 e^{2} v_{F} N_{c}$ or $\approx 8 \mathrm{nH} / \mu \mathrm{m}$ per conducting shell. Thus, the total kinetic inductance for all shells in a CNT bundle is derived as:

$$
L_{k_{t}}=\frac{1}{n_{b} \sum_{i=1}^{N} \frac{1}{L_{k_{i}}}}
$$

where $L_{k_{i}}$ is the kinetic inductance of each shell $i$. Magnetic inductance, $L_{m}$ and mutual inductance $M_{m}$ are also of importance as they can have an impact on dynamic voltage drop behavior. For each shell $L_{m}=\frac{\mu}{2 \pi} \ln (y / d)$ and for a CNT bundle is derived as:

$$
L_{m_{t}}=\frac{1}{n_{b} \sum_{i=1}^{N} \frac{1}{L_{m_{i}}}}
$$

Scalable mutual inductance model between any two shells $i$ and $i+1$ with space distance, $S_{a}$ was presented in [8], [6], [15] and can be estimated as:

$$
M_{m_{i}}=\frac{\mu_{o} l}{\pi} \ln \left(S_{a} /\left(d_{i+1}-d_{i}\right)\right)
$$

and mutual inductance $M_{m m}$, between two CNT bundles with space, $s$ can be similarly expressed as:

$$
M_{m m}=\frac{\mu_{o} l}{\pi} \ln \left(s / d_{\text {out }}\right)
$$

Resistance, capacitance and inductance models for MWCNTs are further utilized to study the dynamic voltage drop and performance latencies for power delivery and signalling interconnects.

\section{THERMAL MOdELING FOR CARBON NANOTUBES}

In an integrated chip, on-chip temperatures may rise well above room temperature level which will impact the behavior of devices and parasitics of interconnects. As interconnect lengths are usually larger than the free mean path of CNTs, there will be electron-phonon scattering along the length of nanotube that would lead to self-heating and temperature rise along the nanotube interconnect. The temperature variations along a nanotube are also dependent on the defect density, alignment and contact resistance. Large contact resistances create large potential barriers at the interfaces of nanotubes for electrons to tunnel through. Additionally, large defect densities cause electrons to localize and conduction happens through thermally activated electron hopping. In literature, there are many contradicting and inconsistent reports on the thermal conductivity and temperature coefficient of resistance (TCR) for carbon nanotubes. TCR is the change in resistance for every 1-Kelvin of temperature rise. In this section, we will exploit the existing physical models of carbon nanotubes and express its electrical properties as a function of temperature.
We investigate both SWCNTs and MWCNTs with various lengths and diameters. We start by deriving the thermal coefficient for resistance for SWCNTS as in [9]:

$$
T C R_{\text {swcnt }}=\frac{\left(L / 10^{3} d_{s}\right) / T_{o}}{1+\left(L / 10^{3} d_{s}\right)\left(T / T_{o}-2\right)}
$$

where $L$ and $d_{s}$ are the length and shell diameter of the nanotube. $T$ is the temperature and $T_{o}=300 K$. For a single wall nanotube with small diameter, the number of conduction channels $N_{c}$ is independent of temperature for long length interconnects (i.e. significantly larger than mean free path at room temperature). Whereas, for large diameter nanotubes with increasing temperature, the number of conduction channels increase which consequently increases $N_{c}$. Similarly, nanotube conductance also depends on nanotube length and temperature. Neutral length $L_{N}$ is the length at which resistance becomes independent of temperature. For lengths smaller than $L_{N}$, the nanotube resistance is mainly influenced by the number of conduction channels, and increasing temperature lowers resistance. Whereas, for lengths larger than $L_{N}$, the mean free path is more important and increasing temperature, increases resistance. Neutral length is derived as in [9]:

$$
L_{N}=\frac{10^{3} a T_{o} d_{s}^{2}}{b+2 a T_{o} d_{s}}
$$

where $a$ is $2.04 \times 10^{-4} \mathrm{~nm}^{-1} \mathrm{~K}^{-1}$ and $b=0.425$. Fig. 2 shows the thermal coefficient of resistance for SWCNTs with different lengths and diameters. We observe that small diameter nanotubes have larger TCR than nanotubes with large diameter. For example, for nanotubes with length $1 \mu \mathrm{m}$ and diameter $1 \mathrm{~nm}$, the TCR $=6.5$ whereas for nanotubes with diameter $10 \mathrm{~nm}$, the $\mathrm{TCR}=4$. For short length and large diameter nanotubes, the TCR is relative small (i.e. $<1$ ), then TCR increases linearly with nanotube length. It is important to note, that TCR is a positive coefficient for SWCNTs with different lengths and diameters.

In Fig. 3, the ratio of resistances with respect to resistance at $\mathrm{T}=300 \mathrm{~K}$ is shown for nanotubes with diameter $1 \mathrm{~nm}$ and various lengths. We note that for short length nanotubes, the resistance ratio is small (i.e. $<1.05$ ) for temperature ranges of $300 \mathrm{~K}$ to $350 \mathrm{~K}$. There is a linear increase to the resistance ratio as the nanotube length increases (i.e. ratio $=1.3$ for $\mathrm{L}=9 \mu \mathrm{m}$ ). Hence, the nanotube resistance increases with temperature but at different rates depending on nanotube length. A similar plot is shown on Fig. 4 where the nanotube diameter is set to $10 \mathrm{~nm}$. We observe a similar trend as in Fig. 3, but the resistance ratios are smaller. This is an important observation for nanotube interconnects where diameter and length of nanotubes can be exploited as a knob for alleviating temperature impact on resistance. For example, the resistance ratio is minor when increasing nanotube diameter from $1 \mathrm{~nm}$ to $10 \mathrm{~nm}$ for nanotube lengths of $10 \mu \mathrm{m}$.

In Fig. 5, the resistance distribution is shown for SWCNTs with different lengths and temperature values for nanotubes of $\mathrm{d}=1 \mathrm{~nm}$. As already mentioned above, for short length nanotubes, the temperature increase does not change the 


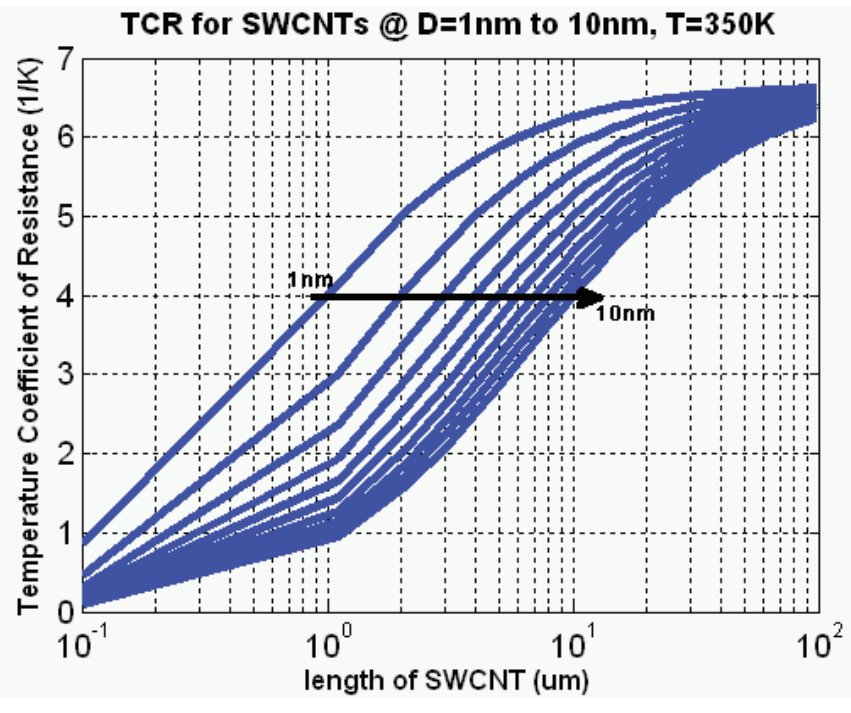

Fig. 2. Temperature coefficient of resistance for SWCNTs.

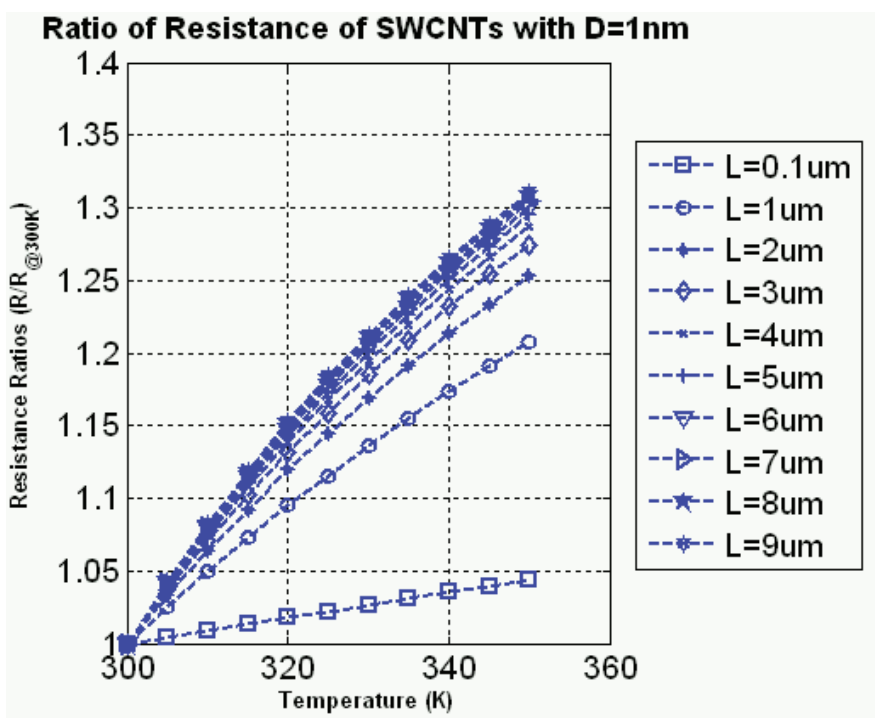

Fig. 3. Ratio of resistances due to temperature impact for SWCNTs with $\mathrm{d}=1 \mathrm{~nm}$.

conduction channels, hence no change in resistance. Whereas, as the length increases, it also increases conduction channels, $N_{c}$ which further increase resistance. Additionally, as temperature increases, we observe an increase on resistance due to temperature impact on $N_{c}$.

To derive TCR for MWCNTs, it is important to understand the heat transport and distribution on individual shells and bundles. Once heat is introduced in the outer shell of MWCNT, the high thermal conductivity along the graphene layer transfer heat at a high flow rate in the circumferential direction, as well as along the tube. Due to close proximity between shells, there is thermal coupling that enables heat flow between shells. Authors in [1] demonstrated that heat introduced at outer shells is evenly distributed to all shells within a short distance, $L \sim$ $50 \mathrm{~nm}$. The total heat flowing through the outer shell is always

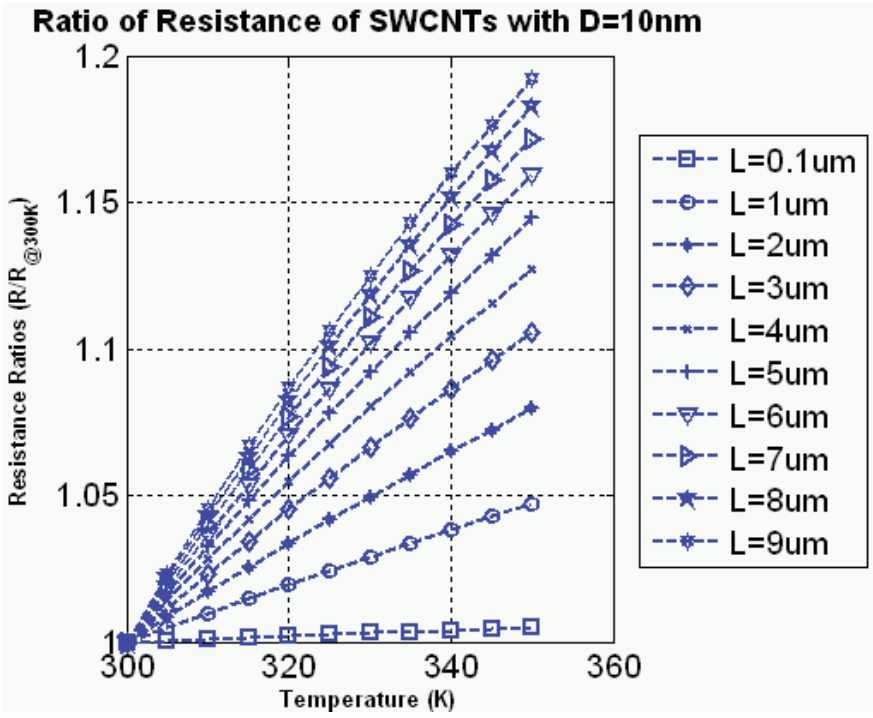

Fig. 4. Ratio of resistances due to temperature impact for SWCNTs with $\mathrm{d}=10 \mathrm{~nm}$.

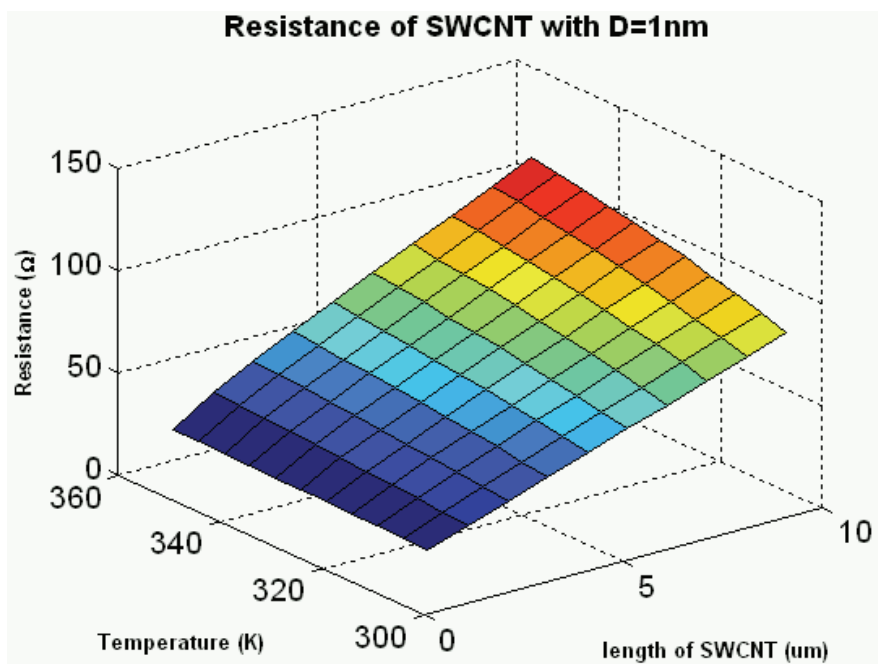

Fig. 5. Resistance distribution for SWCNTs with diameter=1nm for various lengths and temperature values.

higher than the current in the inner shells. We compute the thermal coefficient of resistance based for each shell, which then can be used to compute the resistance of MWCNT with respect to temperature. As the outer shell conducts most of the heat, we derive its TCR as in [9]:

$$
T C R_{\text {mwcnt }}=\frac{\delta R / \delta T}{R_{i_{t h}}}
$$

where

$$
\frac{\delta R}{\delta T}=\left(\frac{R_{q}}{10^{3} d_{\text {out }}}\right)\left(\frac{2 a d_{\text {out }}+b / T_{o}}{\left(a T d_{\text {out }}+b\right)^{2}}\right)\left(L-L_{N}\right)
$$

$R_{i_{t h}}=R_{q}+\left(\frac{R_{q} L}{\lambda_{a c}}+\frac{R_{q} L}{\lambda_{\text {op_abs }}}+\frac{R_{q} L}{\lambda_{\text {op_ems }}^{\text {fld }}}+\frac{R_{q} L}{\lambda_{\text {op_ems }}^{a b s}}\right) / N_{c}$ 


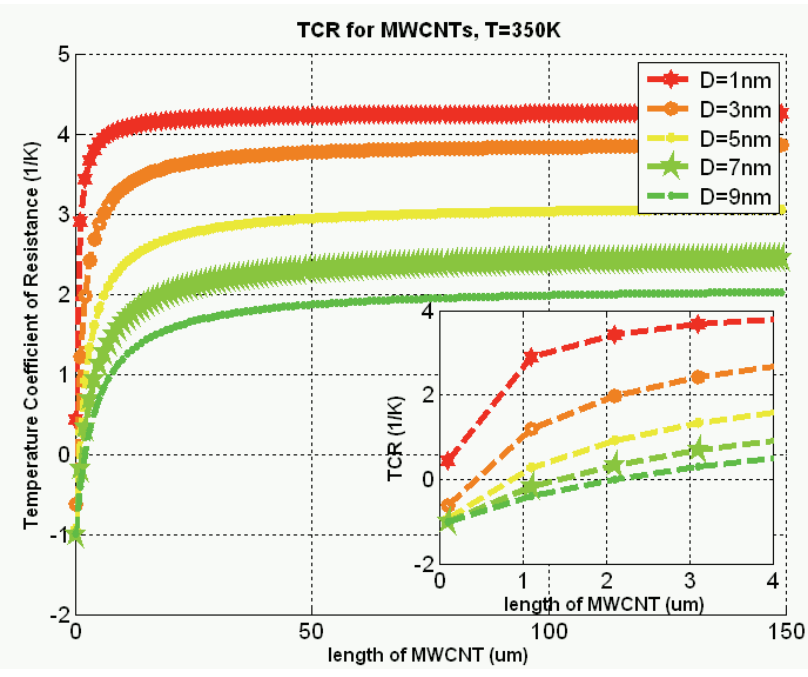

Fig. 6. Temperature coefficient of resistance for MWCNTs for longer lengths (i.e. up to $150 \mu \mathrm{m}$ ) and outer diameters (i.e. $1 \mathrm{n}$ to $10 \mathrm{~nm}$ ). The inset shows the TCR for shorter lengths MWCNTs.

where the mean free path for acoustic scattering is as [4] $\lambda_{a c}=10^{3} d_{\text {out }} T_{1} / T$ where $T_{1}=400 \mathrm{~K}$. Optical phonon scattering can occur if an electron obtains adequate energy (i.e. $\hbar w_{o p} \approx 0.18 \mathrm{eV}$ ), it can emit an optical phonon and get backscattered. The scattering length is (much shorter than acoustic scattering) is computed as $\lambda_{o p}=56 d_{o u t}$ and measured with smaller coefficients (i.e. $\sim 15-20$ )[13]. The mean free path for absorbing an optical phonon is as $\lambda_{o p \_a b s} \approx$ $\lambda_{\text {op }} / n_{\text {phonons }}$ where $n_{\text {phonons }}=1 /\left(e^{\hbar w / K_{B} T}-1\right)$ is the number of phonons and $K_{B}$ Boltzmann constant.

An electron can obtain sufficient energy for emitting an optical phonon either by getting accelerated long enough by electrical field or by absorbing an optical phonon. The scattering lengths can be calculated as:

$$
\lambda_{\text {op_ems }}^{f l d}=\hbar w_{o p} /\left(q V_{\text {bias }} / L\right)+\lambda_{o p}
$$

and

$$
\lambda_{o p \_e m s}^{a b s}=\lambda_{o p \_a b s}+\lambda_{o p}
$$

where $V_{\text {bias }}$ is the applied voltage. It has been shown that increasing the diameter or temperature linearly increases the number of conduction channels in large shells [10]. The average number of channels in a shell can be estimated as

$$
N_{c} \approx \begin{cases}a T d_{s}+b, & d_{\text {out }}>d_{T} / T \\ 2 / 3, & d_{\text {out }}<d_{T} / T\end{cases}
$$

where $d_{T}$ is $1300 \mathrm{~nm} \cdot \mathrm{K}$, whose value is determined by the thermal energy of electrons.

Fig. 6 shows the temperature coefficient of resistance for MWCNTs for various lengths and diameters. The inset figure shows the TCR for shorter length MWCNTs. It is important to note that the TCR for large diameter (i.e. $d_{\text {out }}>1 \mathrm{~nm}$ ) is negative, where the increase in temperature leads to decrease in resistance. This behavior can be explained by Joule heating

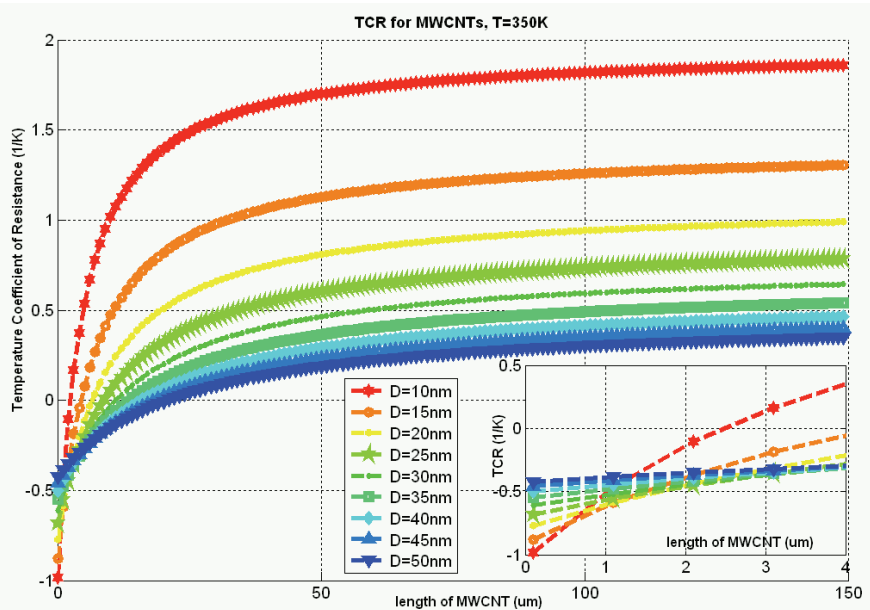

Fig. 7. Temperature coefficient of resistance for MWCNTs for longer lengths (i.e. up to $150 \mu \mathrm{m}$ ) and outer diameters (i.e. $10 \mathrm{~nm}$ to $50 \mathrm{~nm}$ ). The inset shows the TCR for smaller lengths MWCNTs.

(or self-heating) effect and is consistent with negative TCRs obtained by [3], [10]. Theoretical analysis have also shown that negative TCRs for MWCNTs [10], [7]. Negative TCRs can be explained from the fact that there are more channels in MWCNTs contributing to conductance at higher temperatures as per Fermi-Dirac distribution. Larger number of channels lowers both scattering resistance and contact resistance. The negative TCR is opposing with other metals (i.e. copper) conductors, which presents an advantage for carbon nanotubes to be implemented as on-chip interconnect material. We also note that as length of nanotube increases, the TCR also increases till it saturates for lengths longer than $50 \mu \mathrm{m}$.

In Fig. 7, the TCR for large diameter MWCNTs are shown. The diameter varies from $10 \mathrm{~nm}$ to $50 \mathrm{~nm}$ for various nanotube lengths. The inset figure shows the TCR for short length MWCNTs. We note a negative TCR that for large diameter and short length MWCNTs. As length increases, TCR also increases and saturates for MWCNT lengths of $50 \mu \mathrm{m}$. In comparison with Fig. 6, large diameter nanotubes have negative TCRs and small increase in TCR is observed for longer length nanotubes.

From these experiments, we derive that temperature effects can be coped with for interconnects with multi-wall carbon nanotubes where for short lengths even a decrease in resistance can be obtained. Figures 8 and 9 show the resistance distribution for MWCNTs with diameter $10 \mathrm{~nm}$ and $50 \mathrm{~nm}$, respectively. The resistance of MWCNTs with diameter 10nm is at least $1 X$ order of magnitude larger from nanotubes with diameter $\mathrm{d}=50 \mathrm{~nm}$ for $\mathrm{T}=350 \mathrm{~K}$. Such observation is significant for application of CNTs as signalling and power interconnects where large timing errors and voltage drops would be attained. Such large changes in resistance at high temperatures would impact the dimensions of MWCNTs that can be used for building reliable interconnects.

Another important metric is the resitance ratio with respet to resistance at $\mathrm{T}=300 \mathrm{~K}$. Figures 10 and 11 show the resistance 
Resistance of MWCNT with D=10nm

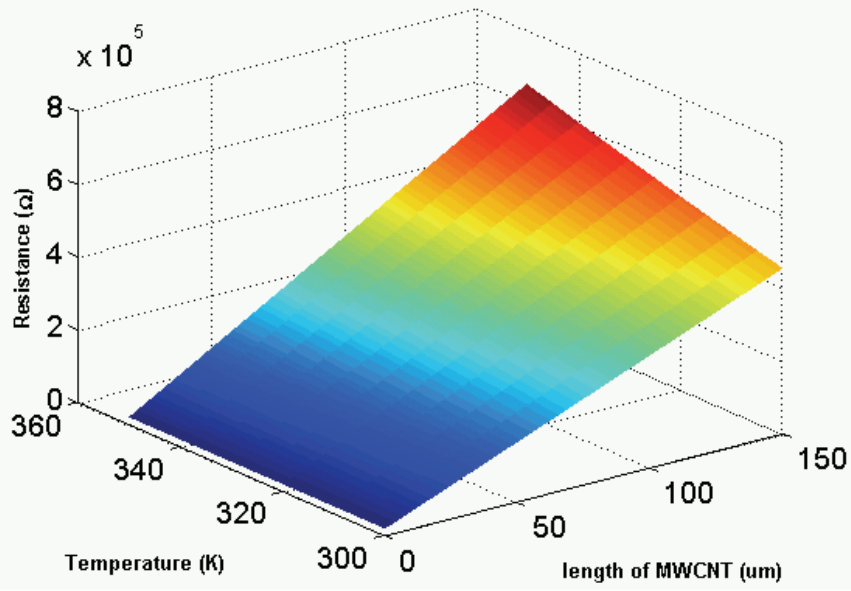

Fig. 8. Resistance distribution for MWCNTs with diameter=10nm for various lengths and temperature values.

\section{Resistance of MWCNT with D=50nm}

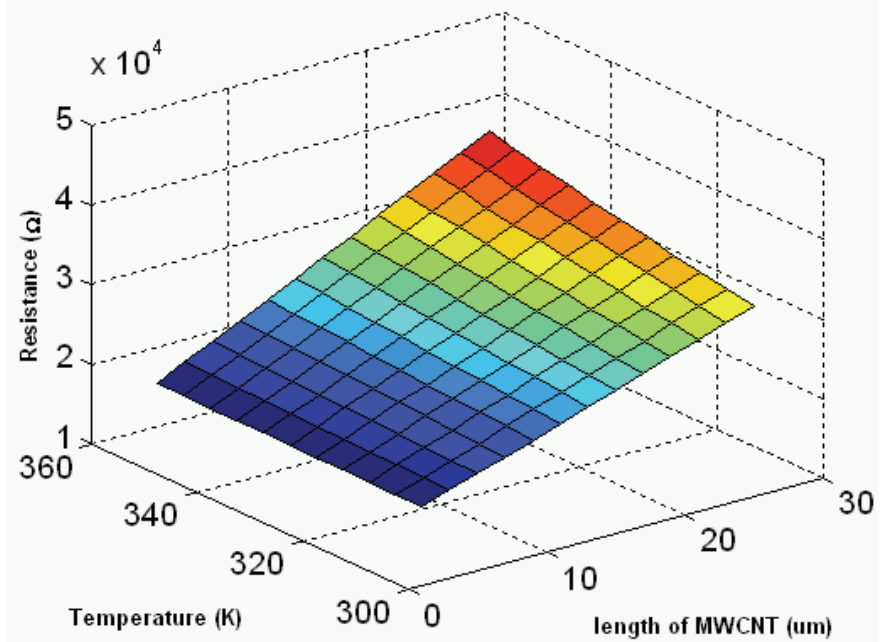

Fig. 9. Resistance distribution for MWCNTs with diameter=50nm for various lengths and temperature values.

ratio for MWCNTs with diameter $10 \mathrm{~nm}$ and $50 \mathrm{~nm}$, respectively. Depending on the diameter and temperature, the neutral length, $L_{N}$ is obtained and also shown in the title of each figure. $L_{N}$ represents the MWCNT length that is independent of temperature. For MWCNT with diameter $d=10 \mathrm{~nm}$, the neutral length is $L_{N}=2.449 \mu \mathrm{m}$. This can also be deduced from the Fig. 10, where resistance ratio is less than 1 for MWCNT lengths of $1 \mu \mathrm{m}$. For MWCNT lengths of $3 \mu \mathrm{m}$, the resistance ratios are almost constant to 1 (i.e. as it is close to $L_{N}$ ). The resistance ratio linearly increases with increase in MWCNT length and reaching maximum of $7 \%$ increase (i.e. maximum resistance ratio 1.07 for $25 \mu \mathrm{m}$ nanotube length).

Fig. 11 shows resistance ratios for $50 \mathrm{~nm}$ diameter nanotubes where $L_{N}=20.6 \mu \mathrm{m}$. Such large $L_{N}$ means that long MWCNT interconnects can used without any dominant impact from temperature. This can also be noted from Fig. 11 where most of

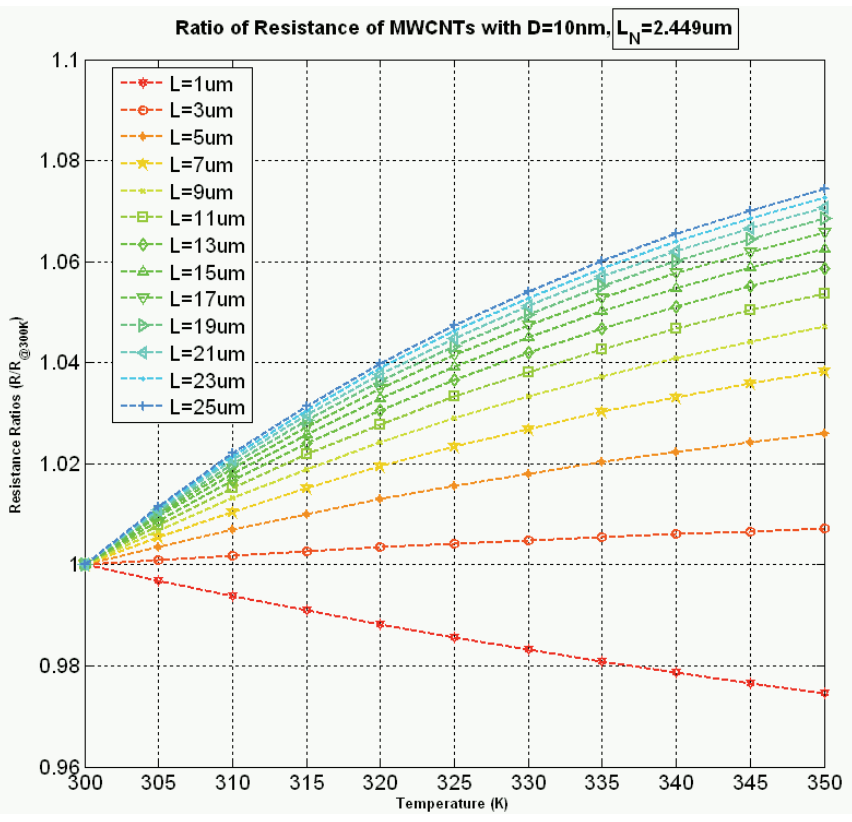

Fig. 10. Resistance ratio for MWCNTs with $\mathrm{D}=10 \mathrm{~nm}$ for various lengths Please note that $L_{N}=2.449 \mu \mathrm{m}$.

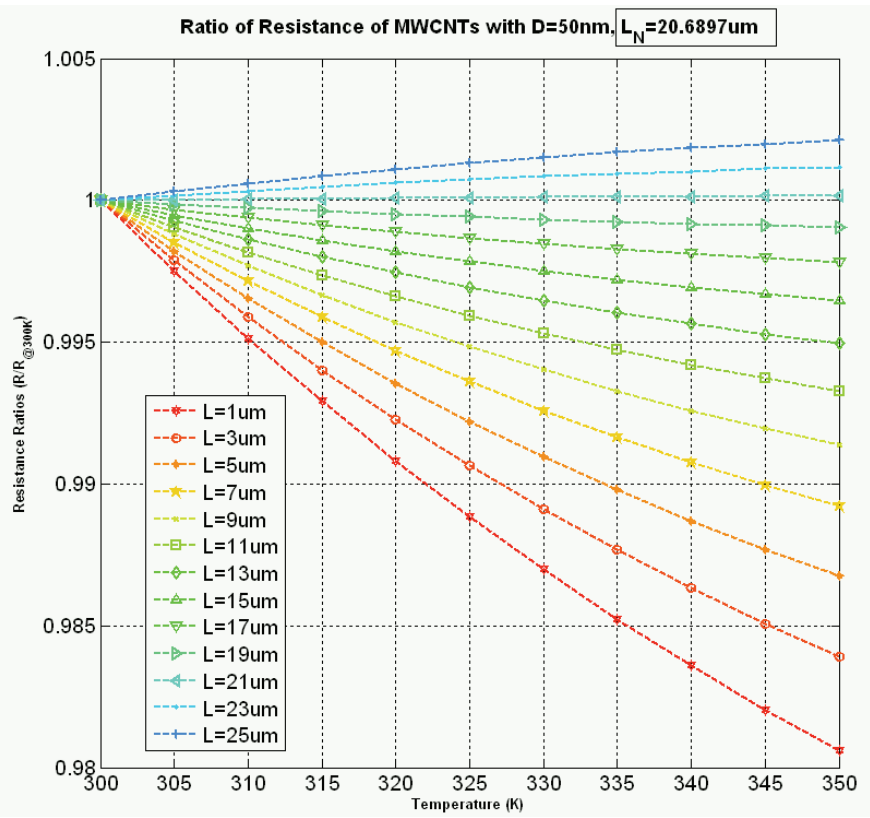

Fig. 11. Resistance ratio for MWCNTs with $\mathrm{D}=50 \mathrm{~nm}$ for various lengths Please note that $L_{N}=20.6897 \mu \mathrm{m}$.

the resistance ratios are below 1. For example, MWCNTs with lengths $\mathrm{L}<L_{N}$ have lower resistance ratio and temperature have a negative impact on the resistance. Such observations are important for selecting interconnect dimensions that are immune to joule heating effects.

To predict the voltage drop on a power delivery network, we analyze a single branch implemented with MWCNTs. To compute voltage drop on the branch, we make assumptions that current flowing on the branch, $I_{\text {branch }}=1 \mu \mathrm{A}, d I_{\text {branch }}=1 \mu \mathrm{A}$, 


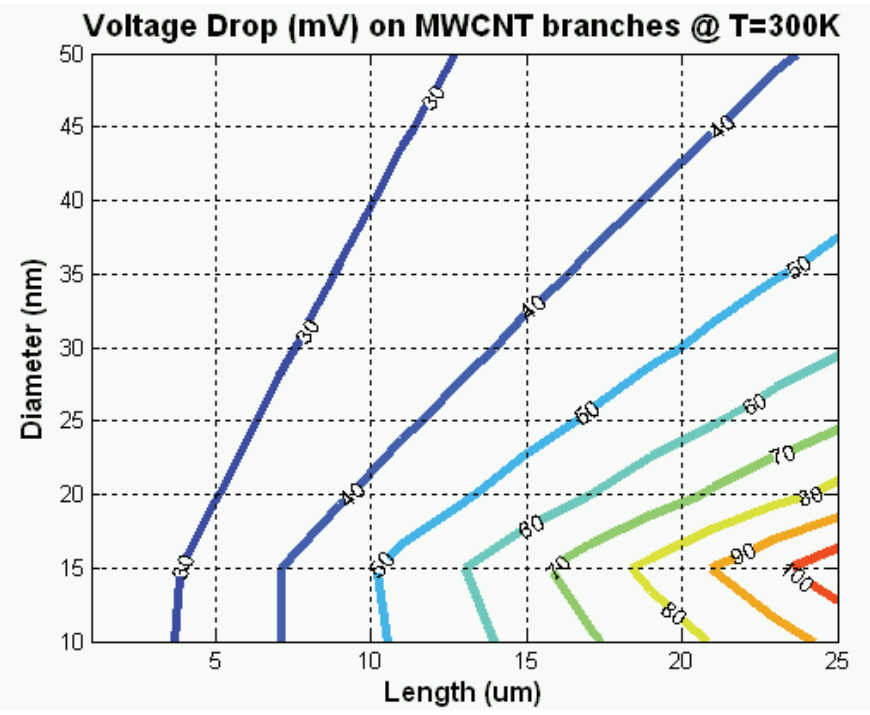

Fig. 12. Voltage drop for MWCNT interconnects with different lengths and diameter for $\mathrm{T}=300 \mathrm{~K}$

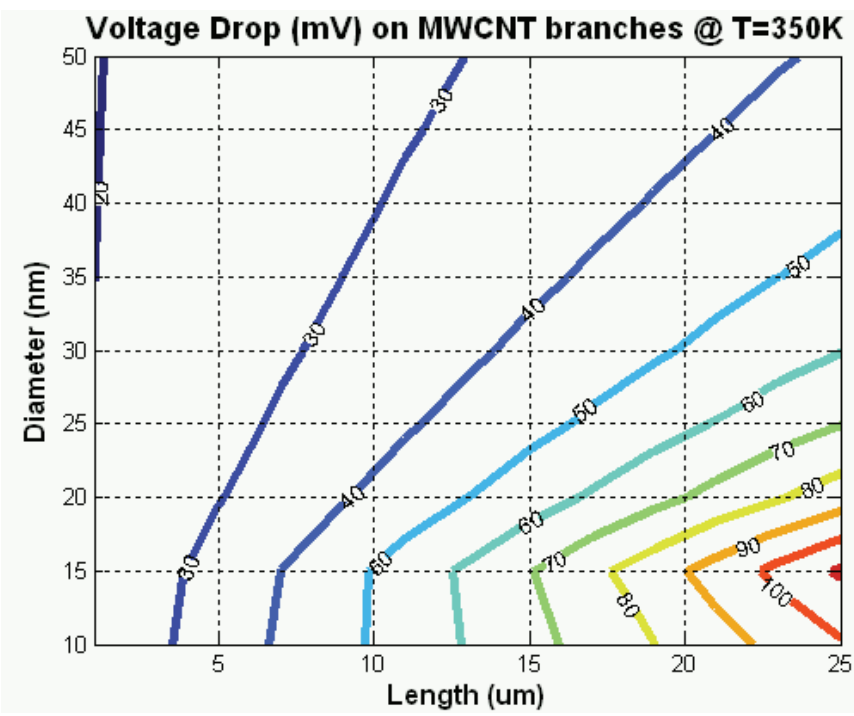

Fig. 13. Voltage drop for MWCNT interconnects with different lengths and diameter for $\mathrm{T}=350 \mathrm{~K}$.

$d t=1 \mathrm{~ns}$ (or $1 \mathrm{GHz}$ switching frequency), and $d V_{\text {branch }}=0.1 \mathrm{~V}$. Please note that these values are simply chosen to quantify branch voltage drop when $1 \mu \mathrm{A}$ current is flowing on the branch for varying MWCNT lengths and diameters. Fig. 12 and 13 show the voltage drop for $\mathrm{T}=300 \mathrm{~K}$ and $\mathrm{T}=350 \mathrm{~K}$, respectively. Overall, we observe minor voltage drop changes due to the impact of negative TCR on resistance. We deduce that selecting MWCNT interconnects length and diameter are essential for limiting the amount of voltage drop. We also compute the delay of MWCNT nanotube interconnects when it is driven by a gate with driver resistance of $200 \Omega$, driver and load capacitances of $0.5 \mathrm{fF}$ and $1 \mathrm{fF}$, respectively. The Elmore

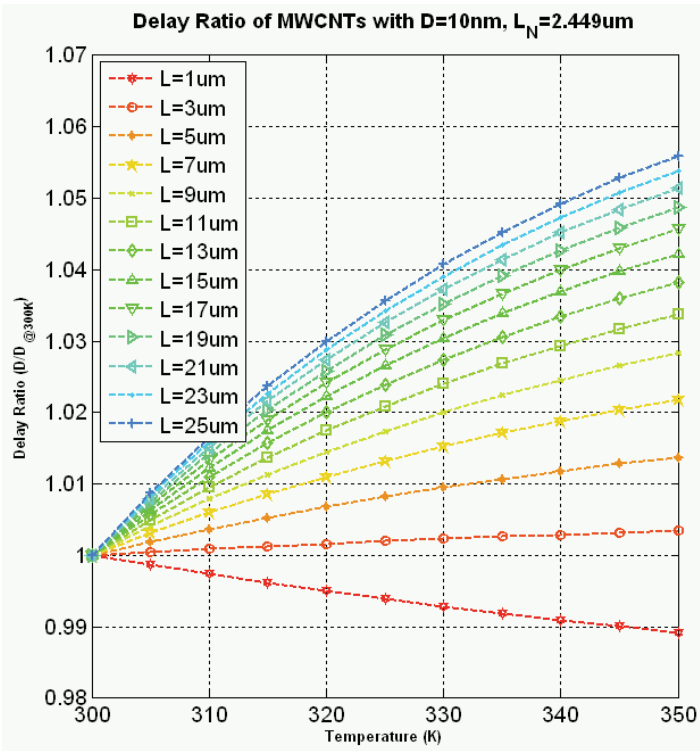

Fig. 14. Delay for MWCNT interconnects with different lengths and diameter $=10 \mathrm{~nm}$.

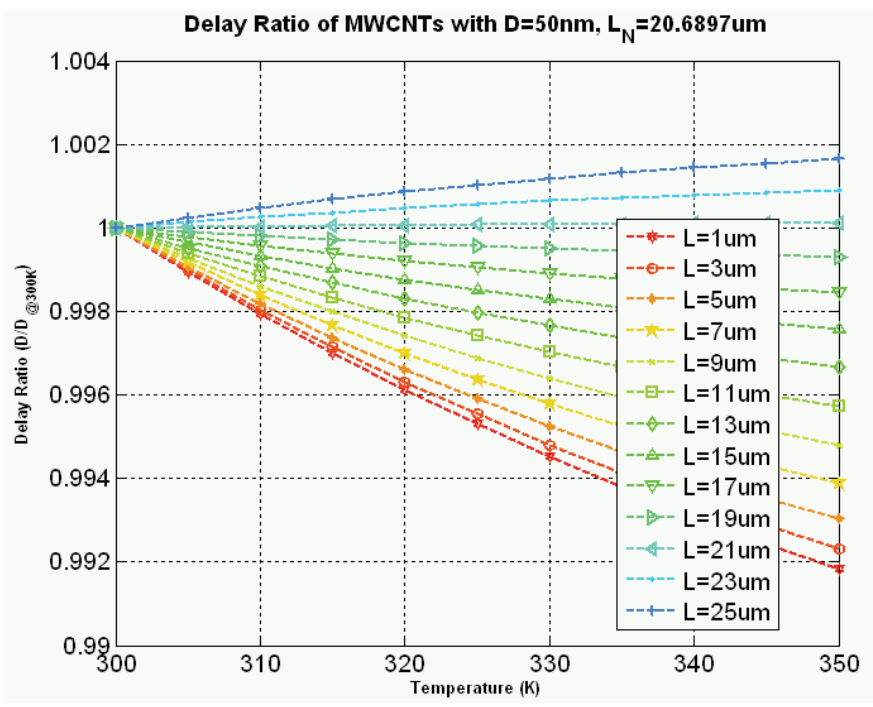

Fig. 15. Delay for MWCNT interconnects with different lengths and diameter $=50 \mathrm{~nm}$.

delay of the segment can be calculated as:

$t_{d}=0.69\left[R_{\text {driver }} C_{\text {driver }}+C_{q}\left(R_{\text {driver }}+R_{i_{t h}}\right)+C_{\text {load }}\left(R_{\text {driver }}+R_{i_{t h}}+R_{c}\right)\right]$

where $R_{c}$ is the contact resistance. Figures 14 and 15 show the delay for MWCNTs with different lengths and temperatures for diameters $\mathrm{d}=10 \mathrm{~nm}$ and $\mathrm{d}=50 \mathrm{~nm}$, respectively. Overall, it is observed a delay increase up to $5.5 \%$ for long length nanotubes with diameter $10 \mathrm{~nm}$, whereas for short length nanotubes a delay speed up is obtained due to negative TCR effect. For nanotubes with large diameter, the changes in delay are minor which points that the nanotube dimensions that are immune to temperature effects. 


\section{Conclusion}

Carbon nanotubes (CNTs) due their unique thermal, and electrical properties are being investigated as promising candidate material for signaling interconnect and power/ground delivery networks. The attractive negative thermal resistance coefficient presents an advantage over other etal conductors for implementing reliable on-chip interconnects. In this paper, we performed a detailed electro-thermal analysis of horizontally and vertically aligned CNTs by investigating the change in resistance due to temperature and its impact on interconnect performance and voltage drop. Analyses demonstrates that CNTs can be efficiently exploited for both signaling and power/ground delivery networks while carefully selecting their diameters and lengths for minimizing the thermal effects.

\section{ACKNOWLEDGMENT}

This project has received funding from the European Union's Horizon 2020 research and innovation programme under grant agreement No. 688612, CONNECT project.

\section{REFERENCES}

[1] A. Aliev, M.H. Lima, E.M. Silverman, and R.H. Baughman. Thermal conductivity of multiwall carbon nanotube sheets: radiation losses and quenching of phonon modes. Nanotechnology, pages 21-035709, 2010.

[2] P.J. Burke. Luttinger liquid theory as a model of the gigahertz electrical properties of carbon nanotubes. Nanotechnology, IEEE Transactions on, 1(3):129-144, Sep 2002.

[3] Alan M. Cassell Franz Kreupl Hong Li, Wei Liu and Kaustav Banerjee. Low-resistivity long-length horizontal carbon nanotube bundles for interconnect applications - part ii characterization. In IEEE Transactions on Electron Devices, volume 60, pages 2870-2876, 2013.

[4] J. Jiang, R. Saito, A. Grüneis, S. G. Chou, Ge. G. Samsonidze, A. Jorio, G. Dresselhaus, and M. S. Dresselhaus. Photoexcited electron relaxation processes in single-wall carbon nanotubes. Phys. Rev. B, 71:045417, Jan 2005.

[5] J.U. Knickerbocker, P.S. Andry, B. Dang, R. R. Horton, C.S. Patel, R.J. Polastre, K. Sakuma, E. S. Sprogis, C.K. Tsang, B.C. Webb, and S.L. Wright. 3d silicon integration. pages 538-543, May 2008.
[6] Hong Li, Wei Liu, AM. Cassell, F. Kreupl, and K. Banerjee. Lowresistivity long-length horizontal carbon nanotube bundles for interconnect applications 2014 - part ii - characterization. Electron Devices, IEEE Transactions on, 60(9):2870-2876, Sept 2013.

[7] Hong Li, N. Srivastava, Jun-Fa Mao, Wen-Yan Yin, and K. Banerjee. Carbon nanotube vias: A reality check. In Electron Devices Meeting, 2007. IEDM 2007. IEEE International, pages 207-210, Dec 2007.

[8] Hong Li, Chuan Xu, N. Srivastava, and K. Banerjee. Carbon nanomaterials for next-generation interconnects and passives: Physics, status, and prospects. Electron Devices, IEEE Transactions on, 56(9):1799-1821, Sept 2009.

[9] A Naeemi, Gang Huang, and J.D. Meindl. Performance modeling for carbon nanotube interconnects in on-chip power distribution. pages 420 428, May 2007.

[10] A Naeemi and J.D. Meindl. Physical modeling of temperature coefficient of resistance for single- and multi-wall carbon nanotube interconnects. pages 135-138, February 2007.

[11] A Naeemi, R. Sarvari, and J.D. Meindl. Performance comparison between carbon nanotube and copper interconnects for gigascale integration (gsi). Electron Device Letters, IEEE, 26(2):84-86, Feb 2005.

[12] A Nieuwoudt and Y. Massoud. Evaluating the impact of resistance in carbon nanotube bundles for vlsi interconnect using diameterdependent modeling techniques. Electron Devices, IEEE Transactions on, 53(10):2460-2466, Oct 2006.

[13] E. Pop, D. Mann, J. Reifenberg, K. Goodson, and Hongjie Dai. Electrothermal transport in metallic single-wall carbon nanotubes for interconnect applications. In Electron Devices Meeting, 2005. IEDM Technical Digest. IEEE International, pages 4 pp.-256, Dec 2005.

[14] L.-C. Qin, X. Zhao, K. Jirahara, Y. Miyamoto, Y. Ando, and S. Iijima. The smallest carbon nanotube. Nature, 408(6808):50, Nov. 2000.

[15] N. Srivastava and K. Banerjee. Performance analysis of carbon nanotube interconnects for vlsi applications. pages 383-390, Nov 2005.

[16] A. Todri-Sanial. Investigation of horizontally aligned carbon nanotubes for efficient power delivery in 3d ics. IEEE 18th Workshop on Signal and Power Integrity (SPI), pages 1-4, 2014.

[17] A. Todri-Sanial, J. Dijon, and A. Maffucci. Carbon nanotube interconnects: Process, design and application. Springer, 2016.

[18] J.W.G. Wildoeer, L.C. Venema, A.C. Rinzler, R.E. Smalley, and C. Dekkler. Electronic structure of atomically resolved carbon nanotubes. Nature, 391(6662):59-61, Jan. 1998.

[19] Lingbo Zhu, Yangyang Sun, Jianwen Xu, Zhuqing Zhang, D.W. Hess, and C.P. Wong. Aligned carbon nanotubes for electrical interconnect and thermal management. pages 44-50 Vol. 1, May 2005. 